\title{
Four co-located antennas for MIMO systems with a low mutual coupling using mode confinement
}

\author{
J. Sarrazin ${ }^{1}$, Y. Mahé ${ }^{1}$, S. Avrillon ${ }^{2}$, and S. Toutain ${ }^{1}$ \\ ${ }^{1}$ IREENA, Université de Nantes, 44306, France \\ ${ }^{2}$ IETR, Université de Rennes I, 35042, France \\ E-mail: julien.sarrazin@univ-nantes.fr
}

\section{Introduction}

Multiple Input Multiple Output (MIMO) systems can drastically improve wireless communication capacity and robustness by exploiting multipath effects. Performances of these multiple antenna systems largely depend on the correlation between received signals. To maximize the capacity, the correlation must be as low as possible. Spatial diversity is usually used to decrease this correlation. However, this requires a space between antennas of about less than $0.5 \lambda$ up to several $\lambda$ (depending on which kind of environment is considered) and it is not always compatible with the limited volume available on a communicant terminal. That is why other kind of diversities are also investigated in order to co-localize antennas still keeping the decorrelation capabilities [1], [2].

Thus, the antenna system presented in this paper is composed of four co-located antennas which are able to produce uncorrelated signals by using both magnitude and polarization diversities. Furthermore, the structure is planar and contains a ground plane which is well-suited to be integrated in a communicant terminal. The diversity potential of the structure is quantify by calculating the envelope correlation between each radiation pattern. The coupling between each access has been decreased by using vias for confinement modes.

\section{Antenna structure}

The antenna system, shown on figure 1, is composed of a dual-polarized $\frac{\lambda}{2}$ microstrip patch and a dual-polarized microstrip square-ring. L-slots have been etched on the patch to reduce its dimensions [3]. So the patch can take place in the ring's middle in order to obtain a compact structure. Because of the slot location, linear polarization orientations of the patch antenna are at $\pm 45^{\circ}$.

Labels $F_{1}$ to $F_{4}$ indicate the coaxial feeding accesses. $F_{1}$ and $F_{3}$ points feed respectively the $\mathrm{TM}_{10}$ and $\mathrm{TM}_{01}$ modes of the patch and $\mathrm{F}_{2}$ and $\mathrm{F}_{4}$ points feed respectively the $\mathrm{TM}_{21}$ and $\mathrm{TM}_{12}$ modes of the square-ring. Thanks to the dualpolarization behavior of the antenna system and since a ring radiates differently from a patch, radiation diversity is achieved between the four feeding access. So the whole structure acts as four independent antennas, like four spaced patches for example.

However, a drawback of such a structure is the strong coupling between patch and square-ring modes, especially between $F_{1}$ and $F_{2}$ and between $F_{3}$ and $F_{4}$. That is 
why metallic vias have been introduced between the square-ring and the patch to enclose modes inside their respective structures. Furthermore, four other metallic vias take place on the square-ring to set short-circuit locations (according to $\mathrm{TM}_{12}$ and $\mathrm{TM}_{21}$ modes) in order to keep its resonance frequency whatever the structure configuration inside the ring (the modified patch).

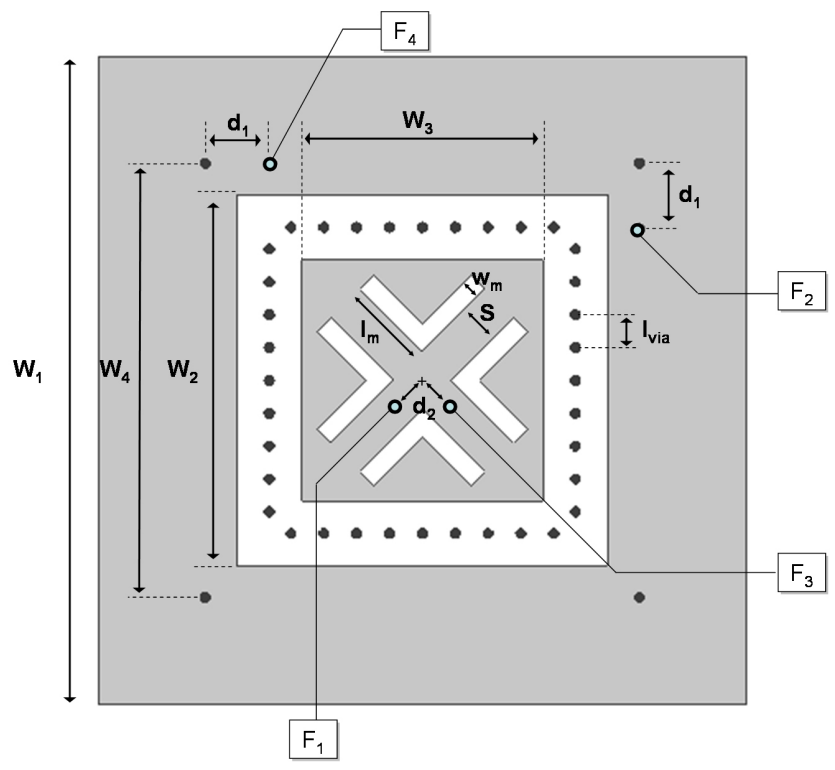

Figure 1: Microstrip antenna configuration.

\section{Via influence}

By enclosing patch and square-ring modes respectively, vias decrease the coupling between them. Via effects on the square-ring impedance can be neglected whereas vias shift slightly the patch resonance frequency. This can be compensated by small variations of the patch length.

The antenna system has been simulated with CST Microwave Studio and the S parameters are represented on figure 2. Curves are drawn for the structure without decoupling via (a) and with decoupling vias (b). Without via, the maximum coupling is about $-16.5 \mathrm{~dB}$ whereas with vias, the coupling is better than $-21 \mathrm{~dB}$. So a $4.5 \mathrm{~dB}$ improvement has been obtained. A parametric study on the via number has been conducted. As expected, the higher the via number, the lower the coupling. Return loss results exhibit a 1.3\% frequency bandwidth for square-ring accesses and $1.8 \%$ for patch accesses around $5.28 \mathrm{GHz}$.

\section{Realization}

The antenna has been build on a Teflon substrate with a relative permittivity $\epsilon_{r}=2.55$ and a thickness $h=1.58 \mathrm{~mm}$ (figure 3). The structure has the following dimensions according to the figure $1: W_{1}=39.5 \mathrm{~mm}, W_{2}=22.6 \mathrm{~mm}, W_{3}=14.7$ $\mathrm{mm}, W_{4}=26.44 \mathrm{~mm}, l_{\text {via }}=2 \mathrm{~mm}, d_{1}=4.5 \mathrm{~mm}, d_{2}=2.3 \mathrm{~mm}, l_{m}=5.4 \mathrm{~mm}$, 


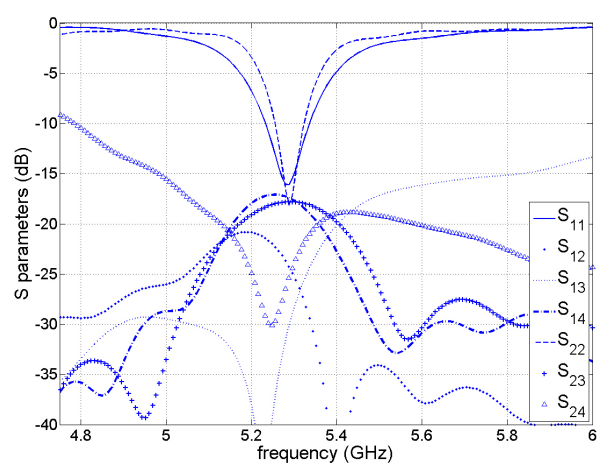

(a)

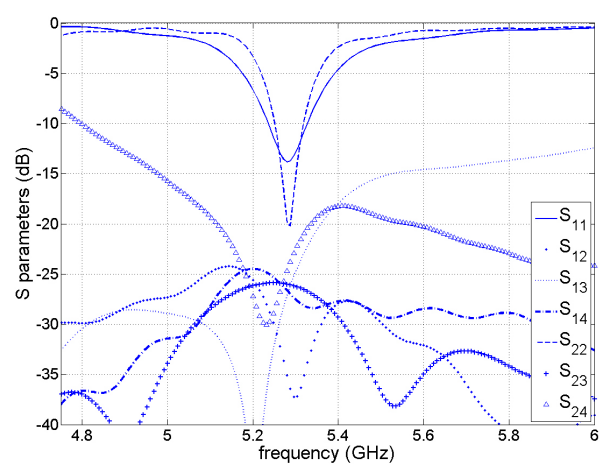

(b)

Figure 2: Simulated S parameters (a) without decoupling via (b) with decoupling vias.

$w_{m}=1.2 \mathrm{~mm}$ and $S=2.5 \mathrm{~mm}$. Central conductor diameters of feeding probe are about $0.6 \mathrm{~mm}$ as well as via diameters.

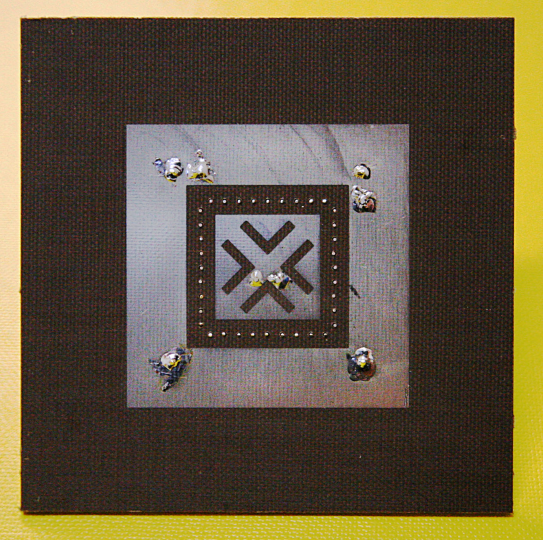

Figure 3: Build antenna.

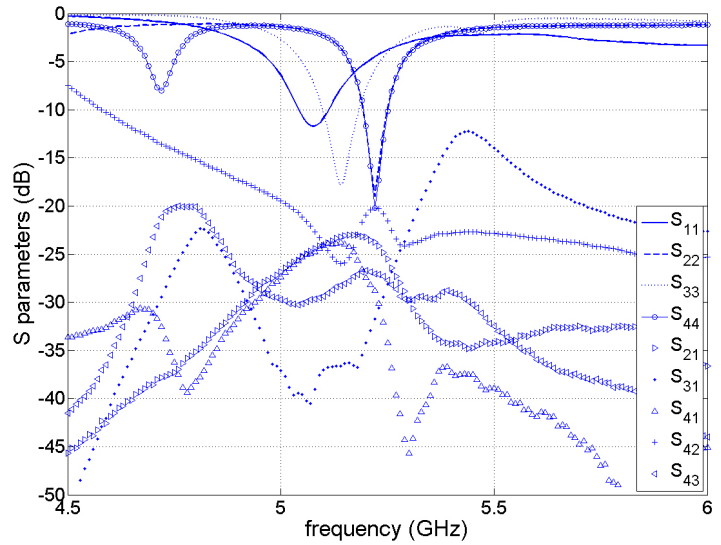

Figure 4: Measured S parameters.

The measured S parameters are shown on figure 4. Curves are in good agreement with the simulation. However, patch resonance frequencies $\left(S_{11}\right.$ and $\left.S_{33}\right)$ are slightly decreased. This is due to a lake of precision during the realization step. Coupling is always better than $-20 \mathrm{~dB}$.

\section{Diversity performance}

An antenna system is well-suited for MIMO applications if many signals can be received with a low correlation. For the proposed antenna system, it depends on the diversity between each access $F_{1}$ to $F_{4}$. In order to quantify this capability, the envelope correlation $\rho_{e}$ between each radiation pattern is calculated with the 
expression 1.

$$
\rho_{e}=\frac{\left|\int_{\Omega}\left(E_{n \theta} E_{m \theta}^{*} p_{\theta}+X E_{n \phi} E_{m \phi}^{*} p_{\phi}\right) d \Omega\right|^{2}}{\int_{\Omega}\left(E_{n \theta} E_{n \theta}^{*} p_{\theta}+X E_{n \phi} E_{n \phi}^{*} p_{\phi}\right) d \Omega \int_{\Omega}\left(E_{m \theta} E_{m \theta}^{*} p_{\theta}+X E_{m \phi} E_{m \phi}^{*} p_{\phi}\right) d \Omega}
$$

$E_{n \theta}$ and $E_{m \theta}$ represent the radiated complex electric fields of accesses $n$ and $m$ along $\theta$ (on spherical coordinates) and $E_{n \phi}$ and $E_{m \phi}$ the fields along $\phi$. The parameter $X$ is the cross-polarization discrimination (XPD) of the incident field and is defined as $X P D=\frac{S_{\phi}}{S_{\theta}}$ (where $S_{\phi}$ and $S_{\theta}$ represent the average power along $\phi$ and $\theta$ ). $p_{\phi}$ and $p_{\theta}$ are the Angle-of-Arrival (AoA) distributions of incoming waves and are assumed to be uniform.

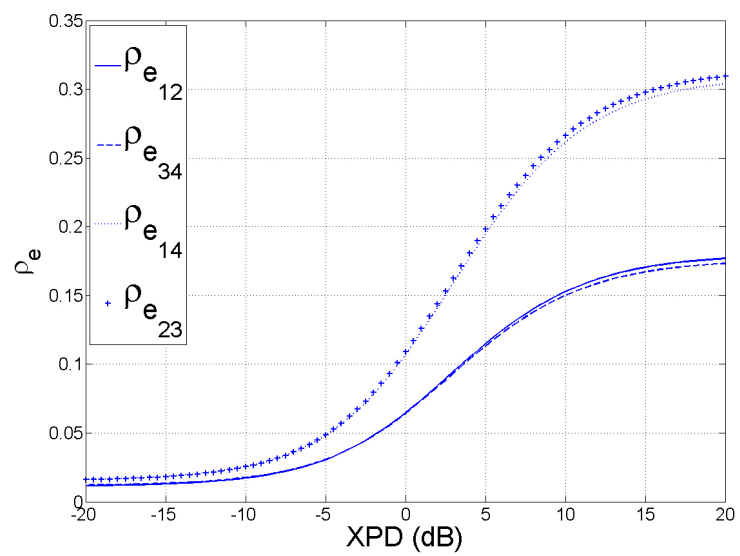

Figure 5: Envelope correlation.

Envelope correlation results are drawn versus XPD on figure 5. Only correlations between the square-ring and the patch are represented. Correlations between each polarization of each structure are not represented because of their very low levels (below $1.10^{-3}$ ). $\quad \rho_{e_{n m}}$ represents the envelope correlation between the radiation pattern of feeding accesses $n$ and $m$. Maximum correlation is about 0.3 which is low enough to make this antenna system well-suited for MIMO applications [4].

\section{References}

[1] A.S. Konanur et Al. , "Increasing wireless channel capacity through MIMO systems employing co-located antennas", IEEE Trans. Microwave Theory and T, vol. 53, n6, pp. 1837-1844, June 2005

[2] C. Waldschmidt and W. Wiesbeck, "Compact wide-band multimode antennas for MIMO and diversity", IEEE Trans. Antennas and Propagation, vol. 52, n8, pp. 1963-1969, Aug. 2004

[3] G.S. Row, S.H. Yeh and K.L. Wong, "Compact dual-polarized microstrip antennas", Microwave Opt. Technol. Lett., vol. 27, pp. 284-287, Nov. 2000

[4] J.N. Pierce and S. Stein, "Multiple diversity with nonindependent fading", Proc. IRE, vol. 48, pp. 89-104, Jan. 1960 